\title{
On the Decay of Bounded Semigroup on the Domain of its Generator
}

\author{
Grigory M. Sklyar
}

Received: 14 November 2013 / Accepted: 13 April 2014 / Published online: 18 September 2014

(C) The Author(s) 2014. This article is published with open access at Springerlink.com

\begin{abstract}
For a bounded $C_{0}$-semigroup on a Banach space $X$, we prove the following statement: the rate of decay of the semigroup on the domain of its generator is bounded by some decreasing function if and only if the spectrum of the semigroup does not contain any pure imaginary points. Our approach is based on the analysis of a special semigroup on the space of bounded linear operators $\mathscr{L}(X, X)$.
\end{abstract}

Keywords Bounded $C_{0}$-semigroup $\cdot$ Rate of decay $\cdot$ Strong asymptotic stability Spectrum · Domain of generator

Mathematics Subject Classifications (2010) 34E99 · 47D03 · 47D06

\section{Introduction}

We begin with recalling the following remarkable result ${ }^{1}$ on asymptotic stability.

Theorem 1 Let $A$ be the generator of a bounded $C_{0}$-semigroup $\left\{e^{A t}\right\}_{t \geq 0}$ on a Banach space $X$ and let intersection of the spectrum $\sigma(A)$ with the imaginary axis $\sigma(A) \cap(i \mathbb{R})$ be at most countable. Then the semigroup $\left\{e^{A t}\right\}_{t \geq 0}$ is strongly asymptotically stable (i.e., $\lim _{t \rightarrow+\infty}\left\|e^{A t} x\right\|=0$ for all $\left.x \in X\right)$ if and only if the adjoint operator $A^{*}$ has no pure imaginary eigenvalues.

This fact was proved by Sklyar and Shirman in 1982 [11] for the case of bounded operator $A$. The method of treating of this problem given in [11] was picked up by Lyubich and

${ }^{1}$ We give an equivalent formulation of the result.

Dedicated to my friend, Professor Nguyen Khoa Son

G. M. Sklyar $(\bowtie)$

Institute of Mathematics, University of Szczecin, Wielkopolska 15, 70-451 Szczecin, Poland

e-mail: sklar@univ.szczecin.pl 
Vu Phong [9] who extended in 1988 the result to the general case. Independently, in 1988, Theorem 1 was obtained by Arendt and Batty [1] who used some different approach.

Let us note that the norm of the semigroup from Theorem 1 may not tend to zero (this occurs if the uniform growth bound $\left.\omega_{0}=\lim _{t \rightarrow+\infty} \log \left\|e^{A t}\right\| / t=0\right)$. In this case, one can see that among the solutions of the abstract Cauchy problem

$$
\begin{cases}\dot{x}=A x(t), & t \geq 0, \\ x(0)=x_{0}, & x_{0} \in X,\end{cases}
$$

given by $x(t)=e^{A t} x_{0}, t \geq 0, x_{0} \in X$, there are some tending to zero arbitrarily slow. At the same time, Batty [2,3] and Vu Phong [10] proved the following result (formulation is taken from [5]).

Theorem 2 Let $\left\{e^{A t}\right\}_{t \geq 0}$ be a bounded $C_{0}$-semigroup on a Banach space $X$ with generator $A$ and

$$
\sigma(A) \cap(i \mathbb{R})=\emptyset .
$$

Then

$$
\left\|e^{A t} A^{-1}\right\| \rightarrow 0, \quad t \rightarrow+\infty
$$

In other words, all the classical solutions of problem (1) (i.e., those for $x_{0} \in \mathscr{D}(A)$ ) tend to zero no slower than a certain decreasing function $g(t)=\left\|T(t) A^{-1}\right\|$, i.e.,

$$
x(t)=e^{A t} x_{0}=\mathscr{O}(g(t)), \quad t \rightarrow \infty, x_{0} \in \mathscr{D}(A) .
$$

The proof of Theorem 2 given in $[2,3]$ is based on the application of some results and methods from [7, 8]. In 2008, Batty and Duyckaerts considered [4] a question of necessity of condition (2) in Theorem 2 for the decay of the norm of the semigroup on the domain $\mathscr{D}(A)$. Their proof is based on careful estimations of some special characteristics of semigroup norm. The main result of [4] in equivalent form is as follows.

Theorem 3 A bounded $C_{0}$-semigroup $\left\{e^{A t}\right\}_{t \geq 0}$ on a Banach space $X$ with generator $A$ satisfies the relation

$$
\left\|e^{A t}(A-\lambda I)^{-1}\right\| \rightarrow 0, \quad t \rightarrow+\infty, \lambda \notin \sigma(A),
$$

if and only if condition (2) holds.

The main goal of the present note is to present another perspective on the subject discussed above, namely we show that Theorem 3 can be proved as a straightforward conclusion of Theorem 1 if we introduce a special semigroup on a space of bounded linear operators, and apply Theorem 1 to this semigroup. Moreover, we observe that our proof of "only if" part of Theorem 3 does not exploit the assumption of boundedness of the semigroup (see Remark 1). Further development of the ideas of this approach will be the topic of a forthcoming paper.

\section{The Space and the Semigroup}

Let $A$ be an infinitesimal operator in a Banach space $X$ and $\left\{e^{A t}\right\}_{t \geq 0}$ be the semigroup generated by $A$. Let us consider the space $\mathscr{L}(X, X)$ of linear-bounded operators from $X$ to 
$X$ and its subspace $Y \subset \mathscr{L}(X, X)$ defined by

$$
Y=\overline{\left\{D R_{\lambda}(A), D \in L(X, X)\right\}}, \quad \lambda \notin \sigma(A),
$$

where $R_{\lambda}(A)=(A-\lambda I)^{-1}$ and $\bar{Q}$ denotes the closure of the linear set $Q$ taken with respect to the norm of $\mathscr{L}(X, X)$. It is clear that $Y$ does not depend on $\lambda$. Next, we introduce an operator semigroup $\{\widetilde{T}(t)\}_{t \geq 0}$ on the space $Y$ given by the formula

$$
\widetilde{T}(t) B=B e^{A t}, \quad B \in Y .
$$

Proposition $1\{\tilde{T}(t)\}_{t \geq 0}$ is a $C_{0}$-semigroup.

Proof Let $B_{0} \in Y$. For any $\varepsilon>0$, we choose an operator $B=D R_{\lambda}(A), D \in \mathscr{L}(X, X)$ such that $\left\|B-B_{0}\right\|<\frac{\varepsilon}{3}$. Then we get the following estimate for $t \in\left[0, t_{0}\right]$ :

$$
\left\|\widetilde{T}(t) B_{0}-B_{0}\right\|=\left\|B_{0}\left(e^{A t}-I\right)\right\| \leq\left\|B\left(e^{A t}-I\right)\right\|+\frac{\varepsilon}{3}\left(1+M_{0}\right),
$$

where $M_{0}$ is $\max _{t \in\left[0, t_{0}\right]}\left\|e^{A t}\right\|$. Using the form of $B$ we obtain

$$
\left\|B\left(e^{A t}-I\right)\right\| \leq\|D\|\left\|R_{\lambda}(A) e^{A t}-R_{\lambda}(A)\right\| .
$$

For any $x \in X$ we have

$$
\left(R_{\lambda}(A) e^{A t}-R_{\lambda}(A)\right) x=\int_{0}^{t} e^{\lambda(t-\tau)}\left(e^{A \tau}+\lambda R_{\lambda}(A)\right) x d \tau
$$

and hence

$$
\left\|R_{\lambda}(A) e^{A t}-R_{\lambda}(A)\right\| \leq M_{1} t_{0}
$$

where $M_{1}=\max _{t, \tau \in\left[0, t_{0}\right]}\left|e^{\lambda(t-\tau)}\right|\left\|e^{A \tau}+\lambda R_{\lambda}(A)\right\|$. Then from (3) we infer

$$
\left\|\tilde{T}(t) B_{0}-B_{0}\right\| \leq\|D\| M_{1} t_{0}+\frac{\varepsilon}{3}\left(1+M_{0}\right) .
$$

Now choosing $t_{0}>0$ such a small number that

$$
\|D\| M_{1} t_{0}<\frac{\varepsilon}{3}, \quad M_{0}<1
$$

we get

$$
\left\|\widetilde{T}(t) B_{0}-B_{0}\right\|<\varepsilon
$$

that means the strong continuity of the semigroup $\widetilde{T}(t)$.

Denote by $\widetilde{A}$ the generator of the semigroup $\{\widetilde{T}(t)\}_{t \geq 0}$. Let $Y_{1} \subset Y$ be the set

$$
Y_{1}=\left\{B R_{\lambda}(A), B \in Y\right\}, \quad \lambda \notin \sigma(A) .
$$

One can see that $Y_{1}$ does not depend on the choice of $\lambda$.

Proposition 2 Operator $\widetilde{A}$ is defined on the set $Y_{1}$ and given there by the formula

$$
\widetilde{A}\left(B R_{\lambda}(A)\right)=B\left(I+\lambda R_{\lambda}(A)\right), \quad B \in Y .
$$

Proof We need to prove the relation

$$
\lim _{t \rightarrow 0} \frac{1}{t}\left[\widetilde{T}(t)\left(B R_{\lambda}(A)\right)-B R_{\lambda}(A)\right]=B\left(I+\lambda R_{\lambda}(A)\right), \quad B \in Y,
$$


where limit on the left hand side of (5) is regarded in the sense of the space $Y$. First, we recall that

$$
\widetilde{T}(t)\left(B R_{\lambda}(A)\right)-B R_{\lambda}(A)=B R_{\lambda}(A)\left(e^{A t}-I\right) .
$$

Using (4) for any $x \in X$, we have

$$
\begin{aligned}
B R_{\lambda}(A)\left(e^{A t}-I\right) x & =B \int_{0}^{t} e^{\lambda(t-\tau)}\left(e^{A \tau}+\lambda R_{\lambda}(A)\right) x d \tau \\
& =\int_{0}^{t} e^{\lambda(t-\tau)}\left(\widetilde{T}(\tau) B+\lambda B R_{\lambda}(A)\right) x d \tau .
\end{aligned}
$$

Since the function $\widetilde{T}(t) B$ is continuous in the norm of $\mathscr{L}(X, X)$ (see Proposition 1) then

$$
\int_{0}^{t} e^{\lambda(t-\tau)} \widetilde{T}(\tau) B x d \tau=\int_{0}^{t} e^{\lambda(t-\tau)} \widetilde{T}(\tau) B d \tau x, \quad x \in X,
$$

and hence we obtain the following operator equality

$$
B R_{\lambda}(A)\left(e^{A t}-I\right)=\int_{0}^{t} e^{\lambda(t-\tau)}\left(\tilde{T}(\tau) B+\lambda B R_{\lambda}(A)\right) d \tau .
$$

From (6), (7) we get

$\frac{1}{t}\left[\widetilde{T}(t)\left(B R_{\lambda}(A)\right)-B R_{\lambda}(A)\right]-B\left(I+\lambda R_{\lambda}(A)\right)=\frac{e^{\lambda t}}{t} \int_{0}^{t} F(\tau) d \tau+\left(e^{\lambda t}-1\right) B\left(I+\lambda R_{\lambda}(A)\right)$,

where

$$
F(\tau)=e^{-\lambda \tau}\left(\widetilde{T}(\tau) B+\lambda B R_{\lambda}(A)\right)-B\left(I+\lambda R_{\lambda}(A)\right)
$$

The function $F(\tau)$ is continuous in the norm of $\mathscr{L}(X, X)$ and $F(0)=0$. Hence $\left\|\int_{0}^{t} F(\tau) d \tau\right\| \leq t \cdot C_{t}$, where $C_{t}=\max _{\tau \in[0, t]}\|F(\tau)\| \rightarrow 0, t \rightarrow 0$, this yields $\frac{e^{\lambda t}}{t} \int_{0}^{t} F(\tau) d \tau \rightarrow 0, t \rightarrow 0$, what implies (5). The proof is complete.

\section{Spectral Properties}

We prove two lemmas on the spectrum of the operator $\widetilde{A}$ defined in the previous section.

Lemma 1 The domain $\mathscr{D}(\widetilde{A})$ of $\widetilde{A}$ is exactly the set $Y_{1}$ and the spectrum $\sigma(\widetilde{A})$ verifies the inclusion

$$
\sigma(\tilde{A}) \subset \sigma(A) .
$$

Proof We already proved Proposition 2 that $Y_{1} \subset \mathscr{D}(A)$. So we need to show the implication:

$$
\text { if } \mathscr{D} \notin Y_{1} \text { then } \mathscr{D} \notin \mathscr{D}(A) \text {. }
$$

To this end, we consider an arbitrary $\lambda \notin \sigma(A)$ and the operator $\widetilde{A}-\lambda I$. We observe that this operator sets a one-to-one correspondence between $Y_{1}$ and $Y$. In fact, if $B \in Y$ or, what is the same, $B R_{\lambda}(A) \in Y_{1}$, then (Proposition 2)

$$
(\widetilde{A}-\lambda I) B R_{\lambda}(A)=B\left(I+\lambda R_{\lambda}(A)\right)-\lambda B R_{\lambda}(A)=B .
$$

So the mapping

$$
B R_{\lambda}(A) \stackrel{\widetilde{A}-\lambda I}{\longrightarrow} B
$$

is one-to one. Besides, this operator has a bounded inverse given by

$$
(\widetilde{A}-\lambda I)^{-1} B=B R_{\lambda}(A), \quad B \in Y .
$$


This means that

1. $\lambda \notin \sigma(\widetilde{A})$;

2. $\mathscr{D}(\tilde{A})=(A-\lambda I)^{-1} Y=Y_{1}$.

The lemma is proved

Lemma 2 Let $\mu \in \mathbb{C}$ be a boundary point of the spectrum $\sigma(A)$. Then $\mu \in \sigma(\widetilde{A})$ and moreover $\mu$ is an eingenvalue of the adjoint operator $\widetilde{A}^{*}$.

Proof For simplicity let us put $\mu=0$. Then from the theorem of boundary point of spectrum $([6,12])$, there exists a sequence $\left\{x_{n}\right\} \subset \mathscr{D}(A)$ such that

1. $\left\|x_{n}\right\|=1, n \in \mathbb{N}$,

2. $\left\|A x_{n}\right\| \rightarrow 0, n \rightarrow \infty$.

Let $\lambda \notin \sigma(A)$. Then $A R_{\lambda}(A) x_{n}=R_{\lambda}(A) A x_{n} \rightarrow 0, n \rightarrow \infty$. This yields

$$
\left\|R_{\lambda}(A) x_{n}\right\|=\left\|\frac{1}{\lambda}\left(A R_{\lambda}(A)-I\right) x_{n}\right\| \rightarrow \frac{1}{|\lambda|}, \quad n \rightarrow \infty .
$$

Let $Y_{2}$ be the image of $\widetilde{A}$ :

$$
Y_{2}=\widetilde{A} \mathscr{D}(\widetilde{A})=\widetilde{A} Y_{1} .
$$

If $D \in Y_{2}$ then (see Proposition 2)

$$
D=B\left(I+\lambda R_{\lambda}(A)\right)=B A R_{\lambda}(A) .
$$

Hence

$$
D x_{n}=B R_{\lambda}(A) A x_{n} \rightarrow 0, \quad n \rightarrow \infty .
$$

Relations (8), (9) imply that

$$
\inf _{D \in Y_{2}}\left\|R_{\lambda}(A)-D\right\| \geq \frac{1}{|\lambda|}>0 .
$$

This means that $R_{\lambda}(A) \notin \overline{Y_{2}}$ and there exists a nonzero functional $f \in Y^{*}$ such that

$$
f(D)=0, \quad D \in Y_{2} \text {. }
$$

This means that

$$
\widetilde{A}^{*} f=0
$$

and the lemma is proved.

\section{Proof of Theorem 3}

Let us assume now that the semigroup $\left\{e^{A t}\right\}_{t \geq 0}$ with generator $A$ is bounded. Let us prove Theorem 3.

Proof Sufficiency. First, we observe that the semigroup, given by the law

$$
\widetilde{T}(t) B=B e^{A t}, \quad B \in Y,
$$

is obviously also bounded. Due to Lemma 1 , we have

$$
\sigma(\widetilde{A}) \cap(i \mathbb{R}) \subset \sigma(A) \cap(i \mathbb{R}),
$$


where $\tilde{A}$ is the generator of $\{\widetilde{T}(t)\}_{t \geq 0}$. So if condition (2) holds, then the set $\sigma(\tilde{A}) \cap(i \mathbb{R})$ is empty. Applying now Theorem 1 for operator $\widetilde{A}$ and semigroup $\widetilde{T}(t)$, we obtain

$$
\widetilde{T}(t) B \rightarrow 0, \quad B \in Y .
$$

In particular, if $B=R_{\lambda}(A)$ we obtain the sufficiency.

Necessity. Assume that condition (2) does not hold, then there exists a boundary point $\mu$ of $\sigma(A)$ with $\operatorname{Re} \mu \geq 0$. Due to Lemma 2 , we conclude that $\mu$ is an eigenvalue of $\widetilde{A}^{*}$, i.e., there exists $f \in Y^{*}, f \neq 0$, such that

$$
\widetilde{T}^{*}(t) f=e^{\mu t} f .
$$

Let $D_{0} \in Y$ be such that $f\left(D_{0}\right) \neq 0$. It is clear that $D_{0}$ can be chosen in the form $D_{0}=$ $B R_{\lambda}(A)$. Then we have

$$
\left(\widetilde{T}^{*}(t) f\right)\left(D_{0}\right)=f\left(\widetilde{T}(t) D_{0}\right)=e^{\mu t} f\left(D_{0}\right) .
$$

Since $\left|e^{\mu t}\right| \geq 1$ and $f\left(D_{0}\right) \neq 0$ this implies that

$$
\widetilde{T}(t) D_{0}=B e^{A t} R_{\lambda}(A) \nrightarrow \rightarrow 0, \quad t \rightarrow+\infty
$$

and therefore

This completes the proof.

$$
\left\|e^{A t} R_{\lambda}(A)\right\| \nrightarrow 0, \quad t \rightarrow+\infty
$$

Remark 1 Let us observe that the proof of necessity in Theorem 3 does not use the boundedness of semigroup $e^{A t}$. That means that the following statement holds.

If $C_{0}$-semigroup $\left\{e^{A t}\right\}_{t \geq 0}$ (not necessarily bounded) on a Banach space $X$ with generator A satisfies the relation

$$
\left\|e^{A t}(A-\lambda I)^{-1}\right\| \rightarrow 0, \quad t \rightarrow+\infty, \lambda \notin \sigma(A),
$$

then

$$
\sigma(A) \subset\{\lambda: \operatorname{Re} \lambda<0\} .
$$

Acknowledgments The author thanks the anonymous referees for valuable remarks and recommendations which have improved the exposition of the article.

Open Access This article is distributed under the terms of the Creative Commons Attribution License which permits any use, distribution, and reproduction in any medium, provided the original author(s) and the source are credited.

\section{References}

1. Arendt, W., Batty, C.J.K.: Tauberian theorems and stability of one parameter semigroups. Trans. Am. Math. Soc. 306, 837-852 (1988)

2. Batty, C.J.K.: Tauberian theorems for the Laplace-Stieltjes transform. Trans. Am. Math. Soc. 322, 783 804 (1990)

3. Batty, C.J.K.: Asymptotic behaviour of semigroups of operators. Functional analysis and operator theory (Warsaw, 1992), vol. 30, pp. 35-52. Banach Center Publ. Polish Acad Sci., Warsaw (1994)

4. Batty, C.J.K., Duyckaerts, T.: Non-uniform stability for bounded semi-groups on Banach space. J. Evol. Equ. 8, 765-780 (2008)

5. Borichev, A., Tomilov, Y.: Optimal polynomial decay of functions and operator semigroups. Math. Ann 347, 455-478 (2010)

6. Daletskiy, Y.L., Krein, M.G.: Stability of solution of differential equations in Banach space. Nauka, Moscow (1970). (in Russian) 
7. Ingham, A.E.: On Wiener's method in Tauberian theorems. Proc. Lond. Math. Soc. 2 38, 458-480 (1935)

8. Korevaar, J.: On Newman's quick way to the prime number theorem. Math. Intell. 4, 108-115 (1982)

9. Lyubich, Y.I., Phong, V.Q.: Asymptotic stability of linear differential equation in Banach space. Stud. Math. 88, 37-42 (1988)

10. Phong, V.Q.: Theorems of Katznelson-Tzaffriri type for semigroups of operators. J. Funct. Anal. 103, 74-84 (1992)

11. Sklyar, G.M., Shirman, V.Y.: On the asymptotic stability of a linear differential equation in a Banach space. Teor. Funkc. Anal. Prilozh. (Kharkov) 37, 127-132 (1982)

12. Sklyar, G.M.: On the maximal asymptotics for linear differential equations in Banach spaces. Taiwan. J. Math. 14, 2203-2217 (2010) 